\title{
Swarm Behavioral Sorting based on Robotic Hardware Variation
}

\author{
Beining Shang, Richard Crowder and Klaus-Peter Zauner \\ Electronics and Computer Science, Faculty of Physical Sciences and Engineering \\ University of Southampton, Southampton, SO17 1BJ \\ $\{b s 3 g 10, r m c, k p z\} @$ soton.ac.uk
}

\begin{abstract}
Keywords: $\quad$ Swarm Robot Simulation, Hardware Variation, Sorting, Behavioral Heterogeneity
Abstract: $\quad$ Swarm robotic systems can offer advantages of robustness, flexibility and scalability, just like social insects. One of the issues that researchers are facing is the hardware variation when implementing real robotic swarms. Identical software can not guarantee identical behaviors among all robots due to hardware differences between swarm members. We propose a novel approach for sorting swarm robots according to their hardware differences. This method is based on the large number of interactions between robots and the environment. Individual robot's unique hardware circumstance determines its unique decision and reaction during each robotic controlling step, and these unique local reactions accumulate and contribute to the robot's global behavior. Accordingly by separating these hardware-triggered global behaviors, swarm robots can be sorted according to their hardware variations.
\end{abstract}

\section{INTRODUCTION}

Swarm robotic research is either simulation-based or hardware-based. In hardware-based swarm robotics, one of the issues' researchers face is the hardware differences between swarm members. Although such differences are very small, identical software in every member of the swarm, does not necessarily result in identical behavior due to the amplification by the non-linear controller, and interactions between the robots and environment.

It has been argued that behaviors of identically trained robots in the same environment are subject to divergence due to hardware variations generated by component manufacture, the assembly process and wear-and-tear (Shang et al., 2013). It was found out through simulated obstacle avoidance experiments (Pugh and Martinoli, 2007) that both genetic algorithms and particle swarm optimization can not withstand different types of sensor variations including offsets and scaling factors. It was further (Elliott and Shadbolt, 2003) argued that although it is very difficult and not cost-effective, identical behaviors of hardware-based robots in a swarm is still possible, where the software is fine-tuned to compensate the inherent hardware differences. The retuning process has to be repeated at regular intervals due to constant wear-and-tear (Roth et al., 1987). Although some researchers have focused on utiliz- ing different hardware equipments to develop swarm robotic systems which are heterogeneous on the hardware level (Dorigo et al., 2013; Parker, 1994), still it can not be ignored that hardware variation always exist and influence robotic behaviors.

In this paper, we propose a novel approach to sort swarm robots based on their behavioral differences which are caused by hardware variations. The sorting of robots is useful in two ways: the efficiency of swarm can be increased by allocating tasks to sorted robots, and expensive calibration process can be avoided for low cost swarm robots by using this self-sorting method.

To achieve the required sorting, we consider the performance characteristics of a large number of simple robots suitable for operating in a swarm, that contain variations of their component parameters. After a large number of interactions with the environment, individual robots shows difference behaviors, it is found that robots with similar behaviors have similar hardware differences.

In Section 2, we discuss the source of hardware variations, and presenting our argument that it is feasible to separating robots against their hardware variations according to their behavioral differences. Following this discussion, we describe the design of the simulation in Section 3 including modeling the robots and testing methods. The results are presented and discussed in Section 4, with the conclusions in Section 5. 


\section{PROBLEM DESCRIPTION}

Irrespective of the manufacturing and assemble processes, real swarm robots come with hardware variations. even if they are built to the same design. Fig 1 illustrated some of the reasons which cause variations at hardware level in in a swarm robot.

An example of components variation would be that the same type of sensors on two robots have different sensitivity (Pugh and Martinoli, 2007). Actuators and batteries can have individual characteristics. For mobile robots, the tires of the wheels are often made from rubber to improve traction, which makes it rather difficult to manufacture with exactly the same diameter (Borenstein, 1996). Furthermore asymmetric load distribution will make tire compress differently, resulting different wheel diameters in practice. In the assembly phase, positions of components and soldering parameters vary. For instance, sensors can be placed with slightly different orientations during soldering, or the quality of soldering joints influence the maximum current, thus the driving ability of the motors varies. In addition, alignment of sensors or wheels may have minor difference. In use, the robots experience differences in wear and tear, including sensor aging, battery draining, mechanic deterioration, or even damage.

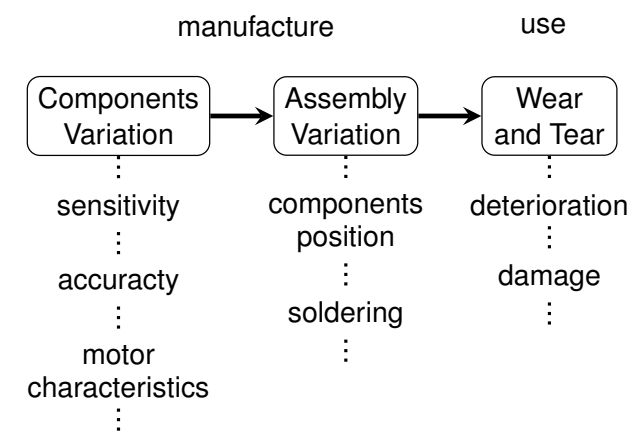

Figure 1: The causes of hardware differences: reasons which can cause robots different in their hardware are listed. They are categorized based on robot's timeline: when robot is manufactured and used. During manufacture, variations exist in components' sensitivity, accuracy, driving ability etc. During assembly, components' placement and soldering parameters varies. When using swarm robots, different damage and deterioration situations are encountered by robots. All of these circumstances are applied to robots' hardware, which differentiate them. This list is not exhausted.

In practice, it is very difficult to get accurate values for each parameter within a complex robotic system. In addition, all measurements have to be taken on each of the robots within the swarm. Furthermore, due to the constant wear and tear, this has to be re- peated at regular intervals. Therefore it is not feasible to modify the performance manually according to their hardware variations. Although it is difficult to get the values for all parameters, the behaviors of the robot is related with its hardware circumstance. Both the decisions and reactions of the robot are always determined by its hardware circumstance. And these decisions and reactions contributes to the robot's global behaviors. Therefore by categorizing robots' behaviors, the sorting of robots according to their hardware variations can be achieved.

\section{SYSTEM MODELING}

As discussed in the previous section, we consider a conventional swarm robot prototype which can follow a reflective line using two IR photoelectric sensors. The modelling of this robot comprises two parts: a model of the abstracted robot hardware and a model of the expected deviation from the ideal prototype.

\subsection{Modeling of the Robot}

The basic features of the robot being simulated are shown in Fig 2, it is a differentially steered, two wheeled robot fitted with casters to the front and rear.

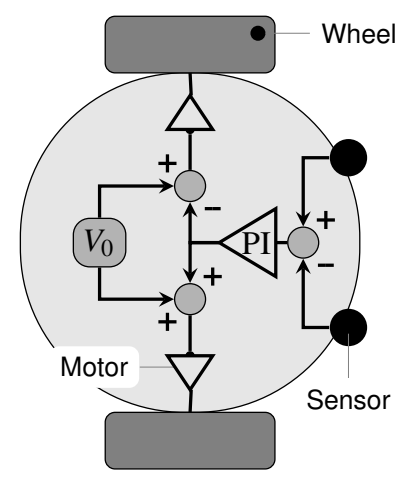

Figure 2: Plan view of the robot and its controller arrangement, showing two sensors in the front and two wheels on each side. The diagram on the robot explains its control mechanism: Voltage difference between outputs of the two IR sensors are amplified by a PI amplifier and then fed to the motors after adding a constant voltage $V_{0}$ which keeps the robot moving forward.

\subsubsection{IR Sensor}

The two IR sensors located at the front of the robot point downwards to measure the reflected light from the surface beneath the robot, including any reflective 
track. The sensor's response (Benet et al., 2002) as voltage is modeled using Eq. 1,

$$
V=\beta+\alpha \int_{0}^{\measuredangle v} \frac{1}{x^{2}} \cos \theta \mathrm{d} \theta
$$

where $\alpha$ is the sensitivity of the sensor, and $\beta$ models the sensor's offset and the effect of ambient light. The sensors response to individual reflective elements within the sensor's viewing angle $\measuredangle v$ is a function of the incidence angle of the reflective light $\theta$ and the distance $x$ between the sensor and the reflecting point (Shang et al., 2013).

\subsubsection{Controller}

The robot uses a conventional PI (proportional and integral) controller to control the robot position along the reflective line. The control algorithm of the robot can be expressed using Eq. 2. The schematic diagram of the controller is illustrated in Fig 2.

$$
\begin{aligned}
\delta(t) & =V_{L s}(t)-V_{R s}(t) \\
V_{\text {out }}(t) & =p \delta(t)+i \int_{-\infty}^{t} \delta(t) \mathrm{d} t \\
V_{L m}(t) & =V_{0}-V_{\text {out }}(t) \\
V_{R m}(t) & =V_{0}+V_{\text {out }}(t)
\end{aligned}
$$

The voltage difference $\delta$ between the left and right IR sensor $V_{L s}, V_{R s}$ is fed to the PI amplifier, the result $V_{\text {out }}$ is used to offset the driving voltages for the left and right motors.

\subsubsection{Drivetrain}

A conventional differential drivetrain system is used. Output voltages from the controller power the left and right motors. Eq. 3 shows the motor model used, which includes any gearbox used.

$$
\begin{aligned}
& \omega_{L}=V_{L m} m_{L} \\
& \omega_{R}=V_{R m} m_{R}
\end{aligned}
$$

where $\omega$ is the angular speed of the wheels. The motors are modeled as pure gain, $m$ between the output of the controller and the wheel speed to simplify the model of the motor and any gearbox, hence.

$$
\left[\begin{array}{c}
\dot{x} \\
\dot{y} \\
\dot{\phi}
\end{array}\right]=\left[\begin{array}{cc}
-\frac{r_{L} \sin \phi}{2} & -\frac{r_{R} \sin \phi}{2} \\
\frac{r_{L} \cos \phi}{2} & \frac{r_{L} \cos \phi}{2} \\
-\frac{r_{L}}{d} & \frac{r_{R}}{d}
\end{array}\right]\left[\begin{array}{l}
\omega_{L} \\
\omega_{R}
\end{array}\right]
$$

where $d$ is the distance between the wheels, and $r_{L}$ and $r_{R}$ are the individual wheel's radius. Based on the wheels' angular speed, the linear change of robotic position $(\dot{x}$ and $\dot{y}$ ) and orientation $(\dot{\phi})$ can be calculated.

\subsection{Modeling of Hardware Variations}

Sensors and actuators can remarkably influence the performance of the swarm robots behavior. Considering each robot as an continuous robotic system, sensors and actuators functions as its input and output. Any tiny difference on these components propagated through each of robot. Thus the robotic behaviors are influenced, and was confirmed in our earlier paper (Shang et al., 2013). Therefore variations simulated in this work are the robot's IR sensors, motor gear boxes and the two wheels.

Variation on IR sensor can normally be found on its performance parameters including sensitivity $\alpha$, viewing angle $\measuredangle v$. In addition, when fitting sensors to the robot, variation can occurs due to misalignment of sensor orientation. In general parameters on which the assembly variation include sensor height $(h)$, sensor lateral offset $\left(O_{l}\right)$ and sensor sagittal offset $\left(O_{s}\right)$. To effectively model the variation found on the motor gearbox and wheel, the following parameters are considered: motor gearbox gain $(m)$, wheel radius $(r)$ and the distance between two wheels $(d)$. In total there are 13 parameters. Apart from wheel separation, the rest of the parameters appear twice as there are two IR sensor and two drive train systems on one robot.

Hardware variation across individual robot in a swarm are different, the values of the same parameter on all the robots follow a Gaussian distribution. In this case, a standard robot was firstly generated with all its parameters values measured according to a real robot. By varying the parameter values for a randomly generated percentage, the rest of its kind were then modeled.

In reality the deviations for the values of the robots' parameters can be different. Therefore three sigma values (large deviation $\sigma=0.05$, medium deviation $\sigma=0.03$, small deviation $\sigma=0.01$ ) are used to generate random number sequences with different distributions. Sensor gain and viewing angle are modeled with small sigma value due to more strict quality control during manufacture. Sensor assembly variation (sensor height, lateral offset and sagittal offset) are comparably small comparing with variations on the motor and gearbox gain, and wheel separation. Details of robotic parameters and the sigma values for Gaussian process are summarized in Table 1. 
Table 1: The parameters of the robot are varied with Gaussian-distributed random numbers which have different distributions. 'L', 'M', 'S' denote for large, medium, small sigma values.

\begin{tabular}{c|c|c|c}
\hline Component & Parameter & Description & Sigma \\
\hline \multirow{4}{*}{ IR sensor } & $\alpha$ & gain & $\mathrm{S}$ \\
& $\measuredangle v$ & view angle & $\mathrm{S}$ \\
& $h$ & height & $\mathrm{M}$ \\
& $O_{l}$ & lateral & $\mathrm{M}$ \\
& $O_{s}$ & sagittal & $\mathrm{M}$ \\
\hline Drivetrain & $m$ & gain & $\mathrm{L}$ \\
\hline Wheel & $d$ & separation & $\mathrm{L}$ \\
\hline
\end{tabular}

\subsection{Training}

A total of approximately 210 different robots were generated and then individually trained using a simple line-following task for the optimal coefficients of the PI controller using exhausted search method. A single period sinusoid reflective line was used, as the robot's ability of both turning right and left would be trained without any bias. During training all robots have the same initial training condition: they all starts with exactly the same position located at the beginning of the sinusoid line with exactly the same orientation. For every robot, this task will be repeated for a number of times to test different sets of controller coefficients. The controller parameters which help the robot achieve the best accuracy in terms of following the target line will be chosen as the optimal parameters.

To evaluate the training results, the trajectory of each robot which is achieved with the optimal parameters is nearly the same with the shape of one period of sinusoid. Therefore, the uniform training method has helps the robots to achieve almost the same trajectories. In other words, the optimal parameters compensate the hardware difference among robots and similar behaviors have been generated.

\subsection{Testing}

After training, robots were then tested in the testing arena illustrated in Fig 3. The testing arena is a long rectangle shaped area. A large number of short reflective lines are drawn in the arena with randomized orientations. All lines sit on the grid, so that they do not overlap each other. The initial condition for all robots are the same: they all start on the left hand side of the arena with the same location, same orientation. While robots were doing the line-following task and interacting with the reflective lines in the arena, the following assumption was made. In the simulation we consider that there is a constant "wind" from the left to the right of the arena, which can influence a robot's speed and orientation. This is analogous to a small sail on top of each robot. If there exists difference between the direction of the wind and orientation of the robot, a force will be applied to the robot by the sail so that the orientation of the robot will change to conform to the wind direction and also a subcomponent of speed to the right will be applied to the robot.

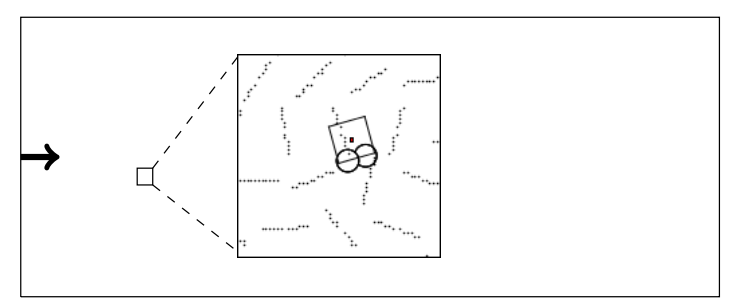

Figure 3: A small square-shape region has been magnified, allowing the length of the line and robot compared. The starting point of each robots and their orientation is denoted by the arrow. The size of the robot is $10 \times 10$ in arbitrary unit. The arenas dimension is in the same unit.

Within the model, if the orientation of the robot and direction of the wind is different, the speed of each robot at each simulation step is the vector addition of the robotic autonomous speed and a subcomponent of speed which is due to the blowing wind, and an angular speed is added to the robot which force the robot to orientate to the right. The reason of making this assumption is that in order to separate all robot as loosely as possible, a tendency of moving to the right of the arena for all robots has to be enforced.

To further reduce the complexity of the problem, all robots will be separated based only on their position on the arena's $\mathrm{x}$ axis. Therefore when the robots go beyond either upper or lower boundary of the arena, it is assumed that they will be picked up and put into the arena from the other side which is the lower or upper boundary accordingly with maintained orientation. In this case, the position of robots with reference to the arena y axis will not matter any more.

Given the size of the arena, robots are able to run in the arena for extended periods of time, thus robots can be separated along the $\mathrm{x}$ axis as loosely as possible.

\section{RESULTS AND DISCUSSION}

Approximately 210 robots have been simulated in two arenas (Arena1 and Arena2). Results are shown in Fig 4 and 5. The only difference between Arena1 and Arena 2 is the orientation of the reflective patterns. According to the results from both arenas, it is found 


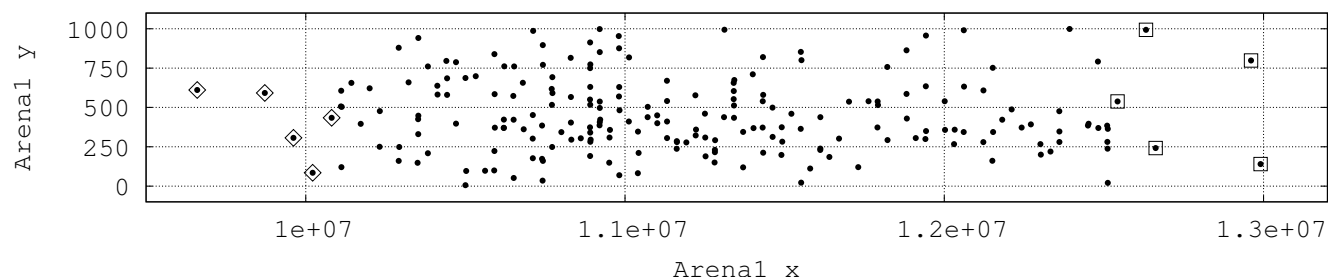

(a) Farthest and Nearest 5 Robots Location in Arena2

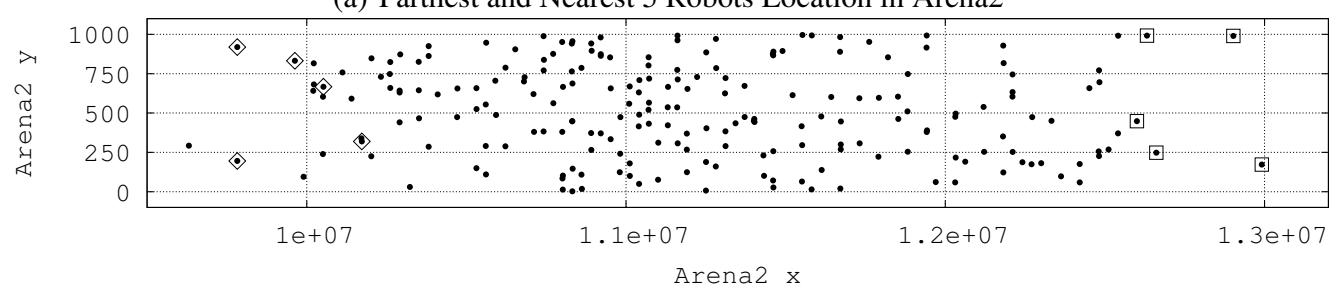

(b) These Robots Location in Arena2

Figure 4: The size of the arenas is in arbitrary unit. Dots in both figures denote the location of robots after the same time. In Fig 4(a), dots with square markers are the fastest five robots in Arena1. These robots also ranked in the top five in Arena2 (square markers) in terms of their end point distance to the starting point. Robots which are near to the starting point in Arena1(marked with diamond) also trail in Arena2(diamond markers).

out that some robots consistently led in the front or left behind in both arenas.

As shown in Fig 5, the rank of robots in terms of $\mathrm{x}$ coordinates for their end points after a fixed simulation period are almost consistent in both arenas. Therefore this approach is able to separate hardwarevaried robots according to their behavioral differences regardless of the orientations of reflective elements in the arenas.

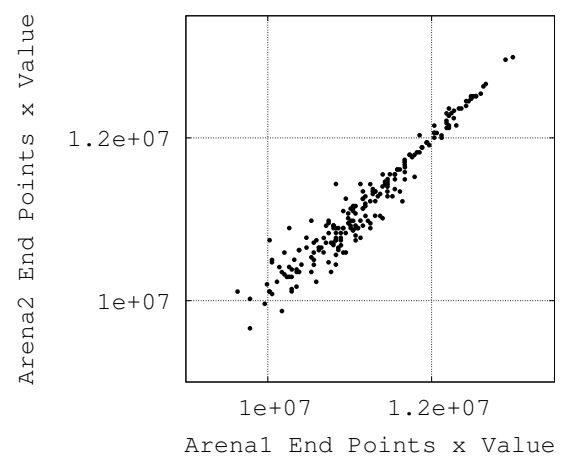

Figure 5: The $\mathrm{x}$ axis values (in arbitrary unit) of the robots' end points in Arenal compared to those in Arena2. Robots which were left behind in Arena1 were also left behind in Arena2. It is the same for robots which run the fastest.

After analysing the data, it is found out that all robots which have travelled far had both strong drive train system on the left and right, in Fig 6. This is consistent with the experiment set-up that the wind blowing effect applied on robots' sail will force robot to orientation to the right. In this case robots with strong left and right motors finish their run the farthest from the starting point.

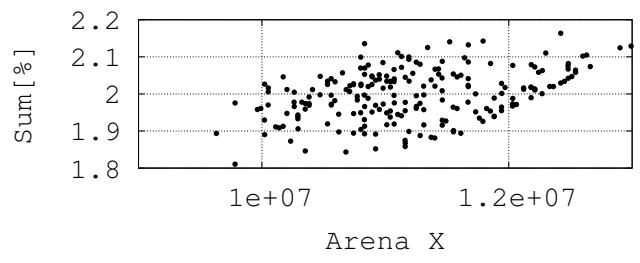

(a) Left and Right Motor Gain Sum

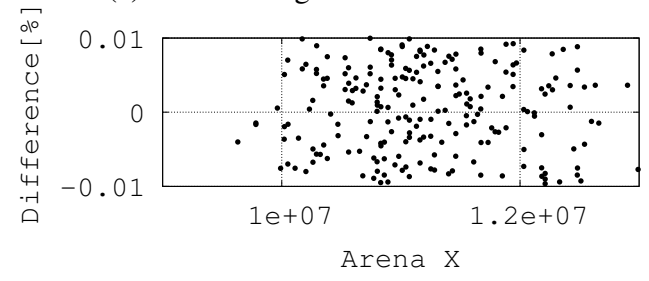

(b) Left and Right Motor Gain Difference

Figure 6: The $\mathrm{x}$ axises of the two figures are in arbitrary unit, the x coordinates of robots' end points in the Arena1 is compared with robots' motor gain variation factor (in percentage). It can be seen from the second figure that for all robots, the gain difference between left and right drivetrain system are very small. From the first figure, all robots which travelled far have large gain on both left and right drive train system.

However strong drive train system is only the necessary condition for large distance between robots' end points and the starting point: not all robots which have large gain on their drive train system travelled far. After analysing the hardware variation factors of all robots with high gain on both their left and right drive train system, there is no clear pattern on other robotic parameters. In other words, the distance between robotic end points and the starting point does 
not depend on one or two parameters of the robots, instead it is determined by the combination of multiple parameters of each robot. To prove this, the robots which end up at both far and near locations have been clustered in the multiple dimensional space of their parameters. In total, each robot has 13 parameters: eight sensor related parameters, three drivetrain system parameters and two parameters for PI controller coefficients. Examples of clustering results are shown in Fig 7. The sorting result shows that there are different parameter settings for robots to travel either far or near from the starting point. The $\mathrm{x}$ values of robotic end points depend on the combination of all parameters instead of individual parameter.

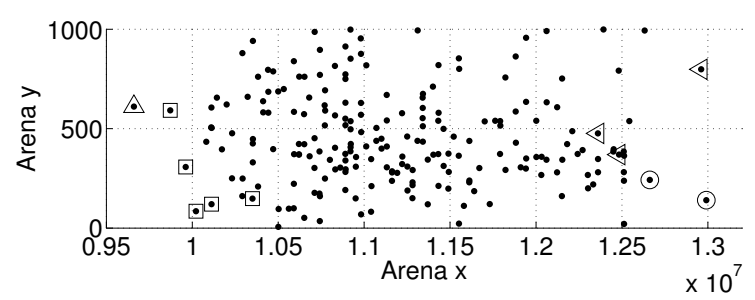

Figure 7: The size of the arena is in arbitrary unit. The end points of the three robots with left-oriented triangles are all very far from the starting point. They are also near to each other in their parameter space. It is the same for robots with circle and square markers. However the end point for the robot marked with upper triangle is near to the starting points and it is not near to any other robots in the parameter space, its unique parameter setting makes it have this behavior

\section{CONCLUSION}

In this work, we propose a novel approach for sorting swarm robot according to their hardware variations. A set of robots is derived from a standard robot by adding minor variations in their parameters to model the intrinsic hardware difference that exists in real robotic swarms and they are then simulated in performing a line following task in the arenas covered with randomly oriented IR-reflective patterns. Results show that this approach is able to sort the group of robots according to their hardware differences. The method of differentiating the robots through the accumulated effect of numerous interactions with the environment is analogous to separating chemical mixtures by chromatography. This method is robust that the sorting of the robots does not depend on the orientations of the reflective patterns in the arena, but on the hardware characteristics of individual robots.
Furthermore it is found out that the behaviors of individual robot is not determined by a single parameter but by the combinations of multiple hardware factors. Different combinations of hardware parameters can help robots achieve similar behaviors.

We view the model system explore the possibility of sorting swarm robots according to their hardware variations in order to tackle the inequality exists in real homogeneous robotic swarms. Future work will focus on improving swarm efficiency by automatically assigning tasks according to their hardwarevariation-triggered behaviors.

\section{ACKNOWLEDGEMENT}

This work was supported by Panzhihua ChangNing Co., Ltd. The authors also acknowledge the use of the IRIDIS High Performance Computing Facility, and associated support services at the University of Southampton, in the completion of this work.

\section{REFERENCES}

Benet, G., Blanes, F., Simó, J., and Pérez, P. (2002). Using Infrared Sensor for Distance Measurement in Mobile Robots. Robotics and Autonomous Systems, 40:255266.

Borenstein, J. (1996). Measurement and Correction of Systematic Odometry Errors in Mobile Robots. IEEE Transactions on Robotics and Automation, 12:6.

Dorigo, M. et al. (2013). Swarmanoid: a novel concept for the study of heterogeneous robotic swarms. In Robotic \& Automation Magazine, IEEE, volume 20, pages 6071.

Elliott, T. and Shadbolt, N. R. (2003). Developmental robotics: Manifesto and application. Philosophical Transactions of the Royal Society of London, Series A, 361:2187-2206.

Parker, L. (1994). Heterogeneous multi-robot cooperation. $\mathrm{PhD}$ thesis, MIT.

Pugh, J. and Martinoli, A. (2007). Parallel Learning in Heterogeneous Multi-Robot Swarms. In IEEE Congress on Evolutionary Computation, pages 3839-3846, Singapore.

Roth, Z., Mooring, B., and Bavani, B. (1987). An Overview of Robot Calibration. IEEE Journal of Robotics and Automation, 3(5):377-385.

Shang, B., Crowder, R., and Zauner, K.-P. (2013). Simulation of Hardware Variations in Swarm Robots. In IEEE International Conference on Systems, Man, and Cybernetics, pages 4066-4071, Manchester. 